\section{The Scanning of Colour and B\&W Film and Photographs for Image Processing, Analysis and Archiving - On a Tight Budget}

\author{
Keith J. Morris \\ Cell Biology Division, The Institute of Ophthalmology, UCL, UK \\ keith.morris@ucl.ac.uk
}

Despite the advent of the new PC based digital camera era, there is still a vast archive of film. The obvious thing to do is to scan film optically using a high resolution film scanner and to convert the image into a digital file for digital distribution, PC archiving, and image processing. The likes of NASA can easily afford the best $\$ 20 \mathrm{k}$ plus PMT drum scanners and pro flatbed scanners for their spaceflight photo archives. See some professional scanners at http://www.imacon.dk, http://www.aztek.com, http://graphics.kodak.com (Creo) and http://www.flatbed-scanner-review.org. You can download selections from the NASA digitised archive at http://grin.hq.nasa.gov.

An optical 8000 dpi Hasselblad Imacon Flextight 848 drum scanner costs around $\$ 15 \mathrm{k}$, with the 949 around $\$ 25 \mathrm{k}$. With drum scanners there is no glass between the detector and the film, unlike that found in professional flatbeds such as the Kodak 'Creo' $\$ 12 \mathrm{k}$ [ $4300 \mathrm{dpi}$ ] IQSmart, the $\$ 45 \mathrm{k}$ [8000 to 14000 dpi] EverSmart Supreme II flatbeds and far cheaper consumer 'photo' flat beds such as the new Epson V750 at \$750. Top end scanners are used by imaging professionals for things like magazine production and museum film archiving. Cheaper dedicated $35 \mathrm{~mm}$ slide and negative scanners also have no glass between the lens and the film surface, although few, other than the $\$ 800$ Epson 3200 [dpi], can scan negatives greater than $9 \times 6 \mathrm{~cm}$, e.g. the 4000 dpi Nikon $35 \mathrm{~mm}$ L50ED at $\$ 500$ and the $\$ 2,5004000$ dpi $35 \mathrm{~mm}$ to 9 x $6 \mathrm{~cm}$ Nikon LS9000 ED film scanner. As well as offering better optics and detectors, higher priced scanners have important adjuncts like attachments to greatly improve the film throughput, essential if you have an archive of $50,000+$ colour slides.

While some of these prices may appear a lot just to scan film, installing a $2 \mathrm{k} \times 2 \mathrm{k}$ or higher resolution digital camera on a transmission electron microscope can easily cost around $£ 20 \mathrm{k}$ to $£ 45 \mathrm{k}$ per TEM system. So many stick with traditional $1 / 4$ plate B\&W TEM developed film negatives. Recently, prices of high resolution 4,000 dots per inch (dpi) consumer flatbed scanners have tumbled, facilitating a cheap way to digitise film for figures in papers or for subsequent image analysis. This has eliminated the need to produce photographic prints from TEM negatives, and allows easy post scan editing and photo-stitching of negatives that would have taken hours in the traditional darkroom.

The black and white (B\&W) silver halide process produces a far more stable image compared to those produced with colour dye substitution (colour slides and negatives). Walt Disney famously created B\&W red, green and blue filter film masters of cartoon films like Snow White to overcome problems associated with colour dye fading. The support material, particularly old celluloid and nitrocellulose stock, may degrade badly with time even as B\&W film, although storage conditions are critical. Modern film uses tough polyester as a base. Early photographers used glass plate as the support medium that was also very durable - until you drop them. Colour fading is a serious problem with colour film - although some colour film processes are more stable than others. Fortunately, much of the original colour can often be restored with digital image processing after scanning. I suppose we should use 'acid free' bag storage to protect our colour negatives, photographs, and slides from atmospheric pollution and decay - just the same as the valuable linen, comic and book owners do (e.g. http://www. savemycomics.com, http://www.conservation-by-design.co.uk ). Modern B\&W TEM film like Kodak 4489 has robust polyester as the support medium and an estimated archive life of about 500 years if stored carefully. Colour film and prints often faded rapidly as the dyes used in earlier film production were far less stable than modern ones.

To prevent our treasured film and photographs being lost to air pollution or damage, the obvious answer is to scan the film into a digitised PC image file (where PC archiving is still an important issue). But how much detail do we need to capture from the original? I will largely ignore photographic prints as any quality $600 \mathrm{dpi}$ reflective flatbed scanner is more than adequate for these. Standard quality $35 \mathrm{~mm}$ colour film is about $6,000 \mathrm{dpi}$. However a high quality prime camera lens can only resolve nearer $4,000 \mathrm{dpi}$, although some extra detail may be seen in the film at $6000 \mathrm{dpi}$. Cheaper consumer zoom lenses or film projectors will do worse than this, often dropping detail to nearer $1,500 \mathrm{dpi}$. Scanning to or above the film's resolution always produces a soft (looks slightly out of focus) digitised image, as the scan sampling now matches or exceeds the films resolution. Assuming a typical film resolution of 120 line per mm (a fairly reasonable number), and that it takes two scanned pixels to represent the line pair, film resolution works out to an equivalent of about 6,000 dpi. Some films claim considerably higher resolving power - 200 lines per mm (lpm) for Acros, for example. Thus, high-end drum scans of 8,000 dpi make sense within that context. The resolving power of Kodachrome II (where two lines can no longer be separated) is $64 \mathrm{lpm}$, thus $30 \mu \mathrm{m}$ structures are the smallest that be resolved with this film.

The line resolution of TEM film is typically better than $200 \mathrm{lpm}$. In TEM it is desirable to maximise electrons for exposure, with slight over-exposure using a very slow film type. In general terms, resolution of a TEM is equal at all magnifications but a low magnification image may require enlarging. Beyond 20x photographic magnification, insufficient electrons have formed the image and "noise" becomes intolerable (Jim Darley, Probing \& Structure Microscopy Supplies \& Accessories). Optimum TEM negative enlargement is about $6 \mathrm{x}$, although up to $10 \mathrm{x}$ to $12 \mathrm{x}$ produces acceptable results with Kodak 4489 , although grain noise from the tissue support resin may become objectionable at high EM magnifications. Optical microscopes have used film for well over 150 years to capture complex magnified images, and have mostly used colour slide film since the 1950 s - the main medium for presentations at the time. In addition, many researchers will have archives of images of such things as laboratory equipment and presentations on $35 \mathrm{~mm}$ film, plus possibly 'Polaroid' or standard photographic prints (with the negatives now lost).

In B\&W film there is usually one layer of silver salts. When the exposed grains are developed, the silver salts are converted to metallic silver, which block light and appear as the black part of the film negative. Colour film uses at least three layers. Dyes added to the silver salts make the crystals sensitive to different colours. Typically, the blue-sensitive layer is on top, followed by the green and red layers. During development, the silver salts are converted to metallic silver, as with black and white film. The by-products of this reaction form coloured dyes. The silver is converted back to silver salts in the bleach step of development and is removed from the film in the fix step, leaving just the coloured dyes. Some films, like Kodacolor II, have as many as 12 emulsion layers, with upwards of 20 different chemicals in each layer (http:// en.wikipedia.org).

As Bob Johnson (http://www. earthboundlight. com/phototips. html) points out, comparing the size of 'photosites' in digital cameras to that of film grain yields some interesting results. The Nikon D2x digital SLR camera has a sensor that measures $23.7 \times 15.7$

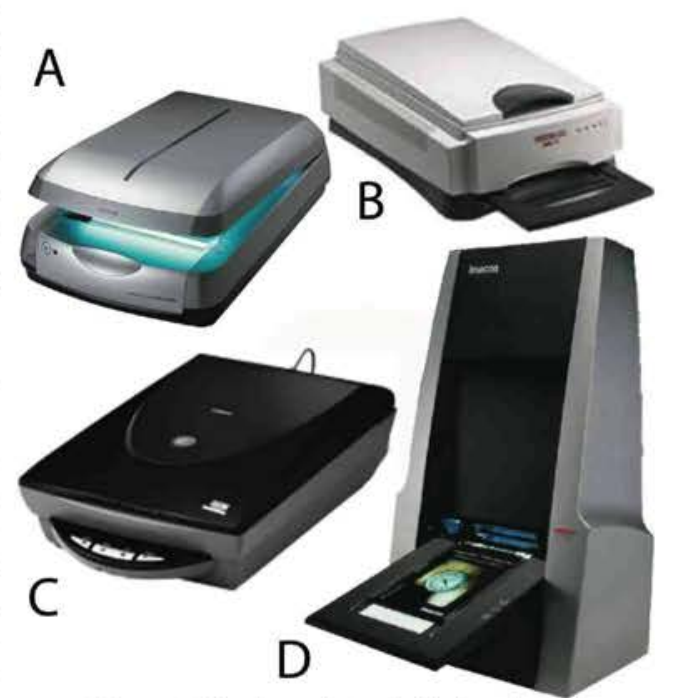

Figure 1. The three photo flatbed and one drum scanners used to produce the images in the other figures. (A) The $\$ 400$ Epson 4990 Photo (B) The $\$ 5,000$ Agfa Duoscan 2550T (C) The \$400 Canon $9950 F(D)$ The $\$ 12,000$ Hasselblad Imacon Flextight 848 drum scanner 


\section{Buehler ViewMet" Metallograph: A Whole New Point of View}

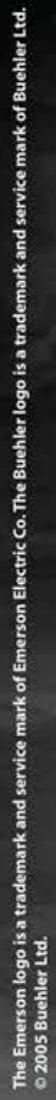

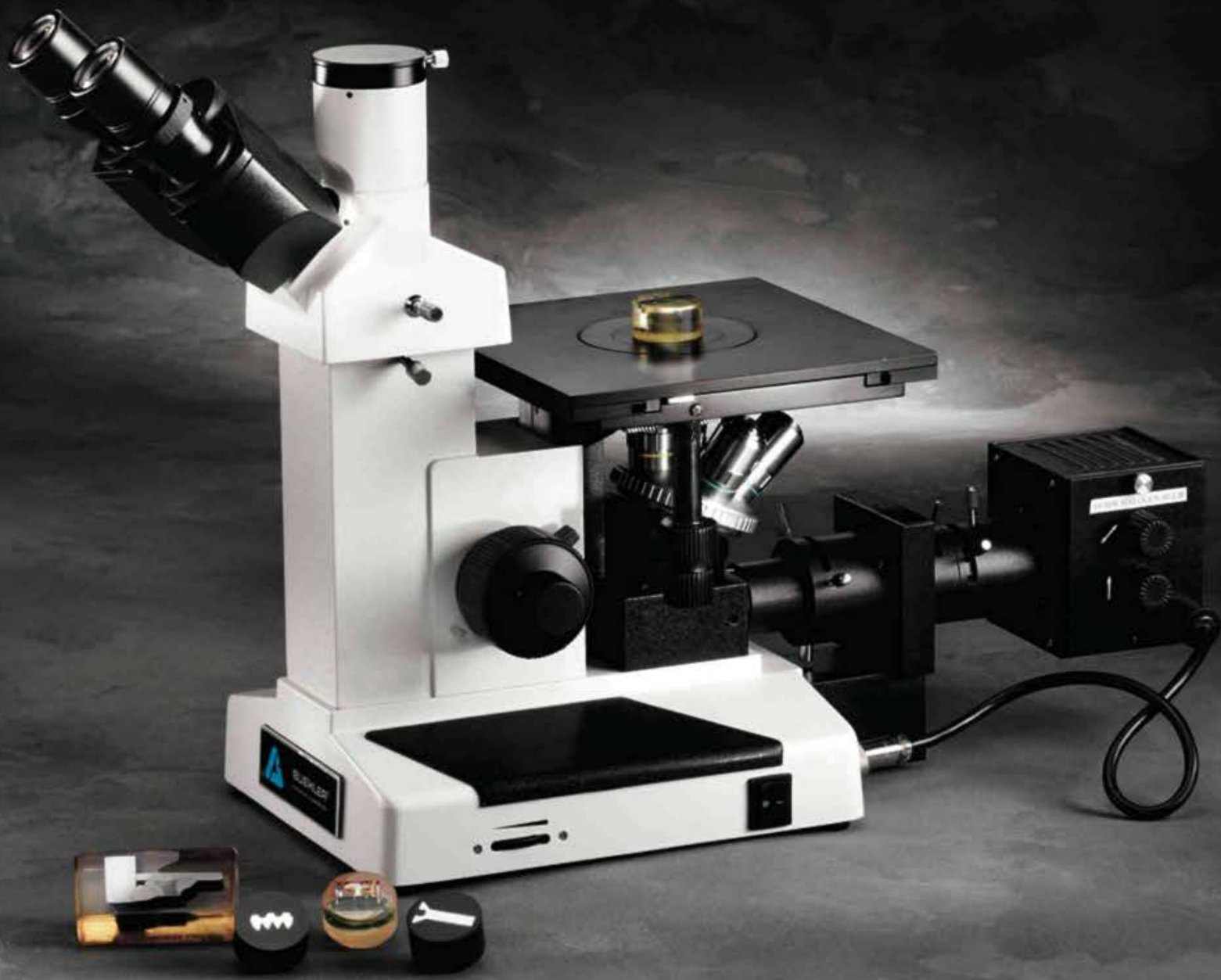

Results are in high demand and time in short supply. A working laboratory metallograph that can provide you with crisp, clear images and ease of use is essential.

Introducing the Buehler ViewMet ${ }^{\mathrm{m} w}$, a dependable inverted bench top microscope, the latest addition to our optical imaging line. This brightfield microscope has the flexibility to accommodate many applications.

- The 5 place nosepiece comes standard with 5, 10,20, and 50X plan-achromat objectives. All objectives $20 \mathrm{X}$ or higher in magnification are spring loaded for durability.

- Enjoy a centerable focusable halogen lamphouse $(6 \mathrm{~V} / 30 \mathrm{~W})$ that provides Köhler illumination

- Conduct quick material checks during sample preparation or take advantage of the robust stage and examine more substantial bar stock sections.

- Generate quantitative results by adding a reticule to the eyepiece. Ensure a superior focused image by adjusting the tension on the coarse focus knob.

- Set up a digital imaging workstation with the trinocular port and included C-Mount

Get a new point of view and keep up with your labs demands.

Worldwide Headquarters Buehler Ltd $\bullet 41$ Waukegan Road Lake Bluff, Illinois $60044 \cdot$ USA Tel: (847) 295-6500 Fax: (847) 295-7979 Email:info@buehler.com Web Site: http://www.buehler.com (An Emerson Industrial Automation subsidiary since 1992)

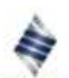

EMERSON Industrial Automation 
$\mathrm{mm}$ (the standard Nikon DX format) and is capable of creating images that are $4288 \times 2848$ pixels ( 12 megapixels total). This gives a pixel pitch of about 5.5 microns. The Nikon D100 SLR has only six megapixels instead of twelve and records images at $3008 \times 2000$ pixels, also on a DX-sized sensor, which works out to about an 8 micron pitch. A digital camera records only red, green or blue at each photosite [in a Bayer mosaic pattern] and interpolates the remaining values. So the true resolution is dependent on the quality of the interpolation algorithm used, as well as the lens and detector. Professional slide film has a grain size of between 8 and 11 microns (Fuji Velvia 100F has an RMS grain size of 9). Photographers who have shot both film and digital end up being surprised how similar the resolution is between the two.

But what about slide and negative film scanners? A Nikon Super Coolscan 4000 is capable of scanning at resolutions up to $4000 \mathrm{dpi}$ (technically spi, or "samples per inch", but everybody calls it dpi). A $24 \times 36 \mathrm{~mm}$ frame of film scanned at 4000 dpi will yield an image of over 20 megapixels. At first glance, this suggests that scanned film images are better than digital camera images, as they have considerably more megapixels. Even a 2700 dpi scanner will give you a nine megapixel file.

Scanners need such high resolution in order to capture all the detail the slide contains. Photographing a scene involves some loss of detail in the translation to photosites or film grain. Any pixel or grain that "sees" a solid colour can accurately record it, but any that need to record half of one and half of another are out of luck. Things are averaged out when something is recorded. Edges get softened and adjacent colours merge since the size of the photosite or film grain determines the smallest detail that can be recorded. If you then scan a frame of film, you subject it to this sort of process a second time, further degrading the image unless you scan at a resolution high enough to resolve the actual grain structure.

So photographers need resolutions of $4,000 \mathrm{dpi}$ and above in a scanner, with sharp focussing, largely for archiving smaller $35 \mathrm{~mm}$ colour slide or negatives. Here, resolving detail in shadow with low noise [i.e. high DMax] is very important - further helped by Photoshop CS's Image, Adjustments, 'shadow/highlight', 'Curves' and 'Brightness/Contrast' applications. DMax in modern consumer photo flatbed scanners such as the \$400 Epson 4990 Photo and Canon 9950F are reported to be around 3.8 to 4.0 (although, like with dpi, manufacturers lie about the true value differently). The pro Imacon Flextight 848 has a quoted DMax of 4.8. Correctly exposed B\&W silver halide negatives have a DMax of nearer 1.5 compared to a colour dye slides 3.5 - so most modern film scanners should easily cope with TEM negatives in terms of dynamic range (http://www.scantips.com). Plus we can only distinguish around 191 grey levels, so 8 -bit (256 greys), rather than 14-bit (16,385 greys) or higher, is mostly fine for $\mathrm{B} \& \mathrm{~W}$ photographs, although scanning $\mathrm{B} \& \mathrm{~W}$ and colour film at higher bit densities may provide more detail. Humans do better

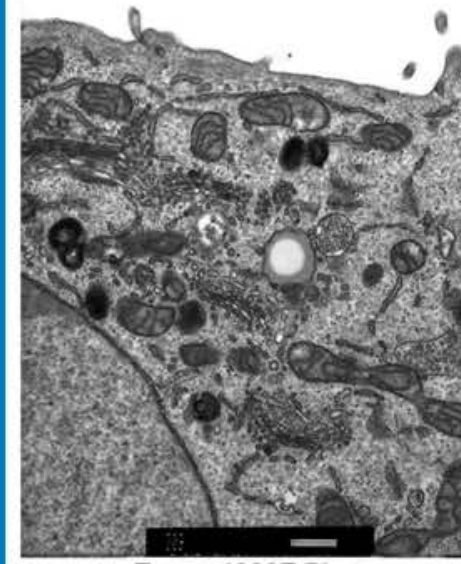

Epson 4990F Photo

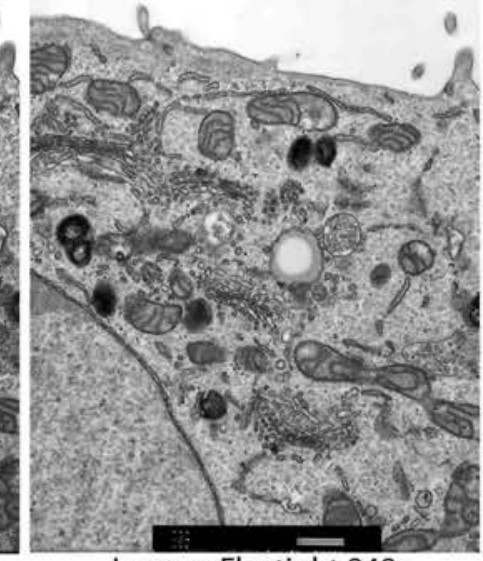

Imacon Flextight 848
Figure 2. A typical high resolution transmission electron microscope (TEM) negative. The negative is Kodak 4489 high resolution electron microscope film - with thanks to Dr. Clare Futter for providing the negative (The Institute of Ophthalmology, London EC1V 9EL, UK). The Epson scan was taken at 4,800 dpi and the Flextight scan was taken at 8,000 dpi. Contrast and brightness was adjusted post-scan in the Epson image to visually match the Flextight 848 one. with colour, plus we perceive colour 'in context' e.g. brown can look yellow (as it is a dark yellow), and so complicated things like CMYK printing and ICC profiling are needed for things like colour film scanning, VDU viewing and printing colours accurately (for details see websites like http://www.tasi. ac.uk). For scanning, twain software applications like Silverfast Ai Studio (www.silverfast.com) can work with Silverfast's own colour IT8 print and film 'targets' to set scan colour correctly. VDU's also need calibrating and brightness/contrast adjustment if you want accurate representation of the scanned digitised image.

As with microscopes, high resolution isn't much use if there's no contrast, but again as with microscopes, increased contrast often reduces resolution. B\&W TEM negatives that initially look good with very high contrast (DMax nearer 2.4) are often inferior in detail to negatives that have a more neutral tonal balance with a DMax nearer 2.0 (you can always increase contrast in Photoshop after scanning). One problem with scanning TEM and optical microscope images on film is that we can't immediately tell if the image is poor after zooming in (particularly if a cheap scanner secretly applies USM or other image processing), whereas with a colour scan of our kids faces, or writing on the side of a ship, it's immediately obvious. For TEM negatives it's easier just to compare the results from different scanners and with the manual view looking at the negative with a light box and an $8 \mathrm{x}$ magnifier. Again for most $35 \mathrm{~mm}$ slide and negatives, $6000 \mathrm{dpi}$ optical is likely to be well beyond the actual resolution of the image on the film. However the secondary process of scanning the camera film through yet another set of optics will further degrade the image quality - the amount of degradation being dependent on scanner quality.

The use of Kodak's (formally Digital) ICE [or Canon's FARE] for dust and scratch removal during film scanning is irrelevant for B\&W negatives as the process is optimised for colour film. Digital ICE is hardware based and pre-scans the film with an infra-red beam. Film is fairly optically transparent at this wavelength, so only the dust and scratches are detected. Software then copies pixels from nearby areas and fills in the areas lost to dust and damage. Thus the 'missing' areas are only cosmetically restored; the original detail is still lost. On scientific images it is probably better to just leave the dust and scratches, particularly as the process further softens the digitised image and can add artefacts. Use a photographic bulb blower to reduce dust on the negative surface. Aerosol air jets work well, but it is very wasteful of the can contents and the propellant can squirt onto the emulsion surface and damage it (even with invertible canisters). Some use brushes as well, but these can scratch the film surface. Digital ICE is great for old home photographs and film, where dust and hairs on faces and scenes can really spoil the image (clone and fill techniques in Photoshop can do this manually, but it really takes time). There are other software solutions such as Kodak's (Digital) SHO, ROC and GEM, that are plug-ins for Photoshop. These can be optimised and integrated into scanners, e.g. the Nikon LS9000ED, but unlike ICE they are still software only. SHO optimises contrast and brightness in a similar manner to Photoshop CS's Shadow/Highlight - particularly good for bringing out detail in shadows (where a high DMax on the scanner also

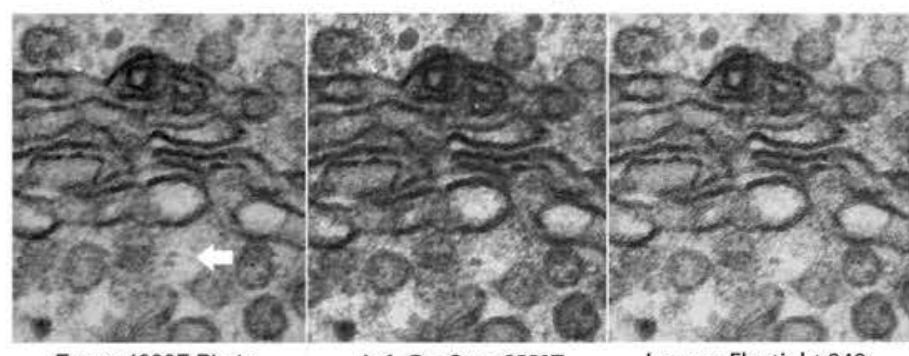

Epson 4990F Photo

Agfa DuoScan 2550T

Imacon Flextight 848

Figure 3. A $100 \%$ enlargement of the same negative scan in figure 2. The image was scanned by the flatbed scanners Epson 4990 Photo and the Agfa Duoscan 2550 T flatbed and the Flextight 848 drum scanner. It is very difficult to see any difference between the image resolution and quality in each scan. Note that there is a minor judder (arrowed) in the Epson 4990 Photo's image scan that would not be noticeable at normal enlargements. This was never seen again, and may be due to vibration or the cheaper scan mechanism. 

helps). ROC adjusts the colour balance for badly faded colour film. GEM reduces image noise and grain from the image and the Airbrush version can 'smooth skin surfaces' by reducing harsh shadows. There are software plug-in versions of ICE to remove dust but these are far less successful than the IR hardware scan and are best avoided. The use of these plug-ins considerably reduces the time taken to do the same in Photoshop. Film grain noise is best minimised by using a high-resolution film scanner of 4000 dpi optical resolution or more.

However, from my experience even going to $£ 5,000$ for a scanner (prices have tumbled recently though), the scan of a negative or slide is often not quite as good as the original if you look at the film using a light box and an $8 \mathrm{x}$ inspection magnifier. I can for instance just make out some of the text of the label on the machine in figure 4 when viewing the original slide, but I can't quite read it when viewing the scanned image. Likewise scanners, particularly consumer flatbeds, will often appear to lose detail in shadows on the scanned image, which can clearly be seen when viewing the original slide - although this shadow detail can be easily restored in Photoshop CS (shadow/highlight). A magnifier and light-box is also very useful to check the colour accuracy of the final scan.

Film grain size optical effects during scanning are also well known - film is made from 'grains' and the more you magnify, the more you can see them. Grain size can also cause optical 'aliasing' effects that degrade the scanned image, so much so that a cheap 400ASA negative colour scan with a 2,700dpi resolution film scanner can produce an appallingly unusable image that image processing can't save - whereas a reflective scan of the $6 \times 4$ " print produces a reasonable A4 image. When going from 2,700 dpi of the old generation of slide/negative scanners up to the 4,000 dpi of modern scanner, many users report far better image quality, and put it down to reduced effects from grain aliasing. At these resolutions, film grain is still very apparent during enlargement, as much $35 \mathrm{~mm}$ film grain has a lower dpi than the scanners, but 'aliasing' artefacts from the grain are greatly reduced.

In practice, many problems in scanned image quality are as much due to the ease of magnifying a digital image compared to the film - a few clicks and you have a 'print' the size of a wall. Also the original image quality on film will be entirely dependent on the quality of the camera prime lens optics and the film originally used. Modern film resolution can vary from 80 to $200 \mathrm{lpm}$, and specialist film can go up to $320 \mathrm{lpm}$ or more. Generally B\&W is capable of the best resolutions, while increase in film speed (ASA) reduces resolution by increasing grain size to improve light sensitively. Likewise, ignoring camera shake, reducing a camera lens aperture from $f-2.8$ to $f-22$ can reduce the maximum theoretical resolution of the lens due to diffraction, although film resolution itself will rise as you stop down, as optical aberrations will reduce ( $f$-stops 8 to 16 being optimal). Naturally if you want to zoom in on a negative, it would have been better to do this on the electron or optical microscope and take another picture at higher magnification instead.

Our eyes prefer a fuzzy analogue gradation of colour to the much defined little squares of a pixellated image at the same resolution, plus we are quite good at discerning contrast. Also digital camera's often do some intense image processing during image capture (e.g. noise reduction, colour correction and sharpen) so you have to work on the image after scanning to get the same result. Surprisingly, twain software can also have an effect on scanned quality and cheaper scanners may benefit from using Silverfast over the bundled scan software. Given the high cost of a Creo pro flatbed or Imacon type drum scanner you can pay someone to scan a few important negatives or slides at $8,000 \mathrm{dpi}$ on their scanner and this is typically $\$ 20$ per picture depending on resulting image file size.

You can get pretty good results from the new breed of $\$ 500$ flatbed film scanners though, particularly with large format negatives. See websites like http://www.photo-i.co.uk for reviews of the Canon 9950F and the similar Epson 4990 Photo. At the time of writing the latest $\$ 700$ Epson V700 and V750 Pro look to have the crown as the best prosumer photo flatbeds. So how do the likes of these new breed of cheap scanners compare with a $\$ 5,000$ Agfa Duoscan flatbed scanner bought in 2000, or a Imacon Flextight 848 costing $\$ 12,000$ ?

If you keep the negative or slide anyway, being able to print to A3 size is adequate for most purposes. People will choose their scanner largely on cost, particularly home users. At home I use the cheap Canon $9950 \mathrm{~F}$ flatbed (c2005) that has replaced my 2,700 dpi SCSI Scanwit 2740s (c2002) dedicated slide scanner - it's faster and easier to use with USB2/Firewire. At work, we have an Apple-based \$5,000 Agfa Duoscan T2550 (c2000) and a cheap \$400 Epson $9950 \mathrm{~F}$ photo (c2006). I have scanned a series of TEM negatives that were made this year using Kodak Electron Microscope Film 4489, size 3.25" $\mathrm{x} 4$ " $(8.3 \times 10.2 \mathrm{~cm})$. Kodak 4489 probably has a resolution of more than 200 $\mathrm{lpm}$ with its 'ultra-fine grain size. In addition I scanned a few $35 \mathrm{~mm}$ Agfachrome CS colour reversal film (ASA around 100) taken in the late 1970's as part of my $\mathrm{PhD}$ studies. The Agfachrome will probably have a resolution of about $120 \mathrm{lpm}$, and be typical of the type of old $35 \mathrm{~mm}$ slides most will want to scan. These Agfachrome CS slides haven't shown any noticeable colour fading over the last thirty years. Note that $35 \mathrm{~mm}$ colour slide scans often produce far better scan results than that with cheap $35 \mathrm{~mm}$ colour negatives. This is because film negatives were exclusively manufactured for photo printing, and so weren't designed to be enlarged much beyond A4, whereas slide film was routinely enlarged beyond this during projection. Many consumer colour negatives also have higher ASA's of 200 to 400, with its increased grain size, that made them more suitable for cheaper hand held cameras. So $35 \mathrm{~mm}$ colour negatives don't scan so well and will require more Photoshop post-editing as well. Again, as with colour slides, Silverfast IT8 targets are needed to set the scan colour accurately.

Figure 1 shows the scanners used to scan these films. Figures 2 and 3 show scanned B\&W images from Kodak TEM 4489 negative film. Figures 4 and 5 show scanned images of the Agfachrome CS $35 \mathrm{~mm}$ colour slides. The image quality of all the flatbed scans are a little out of focus (i.e. 'soft') compared to the Flextight 848 drum scanner at full magnification, but the careful use of USM (unsharp mask) in Photoshop can improve this somewhat. But they are fine up to $\mathrm{A} 3$ printing at least (from a $35 \mathrm{~mm}$ slide). Flatbed scans need more twain tweaking and post scan editing than dedicated film and drum scanners. Leave things like USM and colour balance to Photoshop where you have far more control, but use the twain interface to set things like brightness in dark negatives and dust ICE/FARE removal.

Rather than go into a long discussion on the quality of the scans, the figures clearly show that all the scanners, whether costing $\$ 400$ or $\$ 14,000$, all produce output that is very difficult to tell apart. The Flextight scans have no Photoshop post-editing. With colour film, the cheap Canon 4490F scans needed the Photoshop CS shadow/highlight utility to bring out the shadow detail correctly, otherwise it would have been far too dark. The Flextight resolved shadow detail marginally better than the Canon, and with less noise. The colour balance was more accurate with the Flextight scan - the scanner

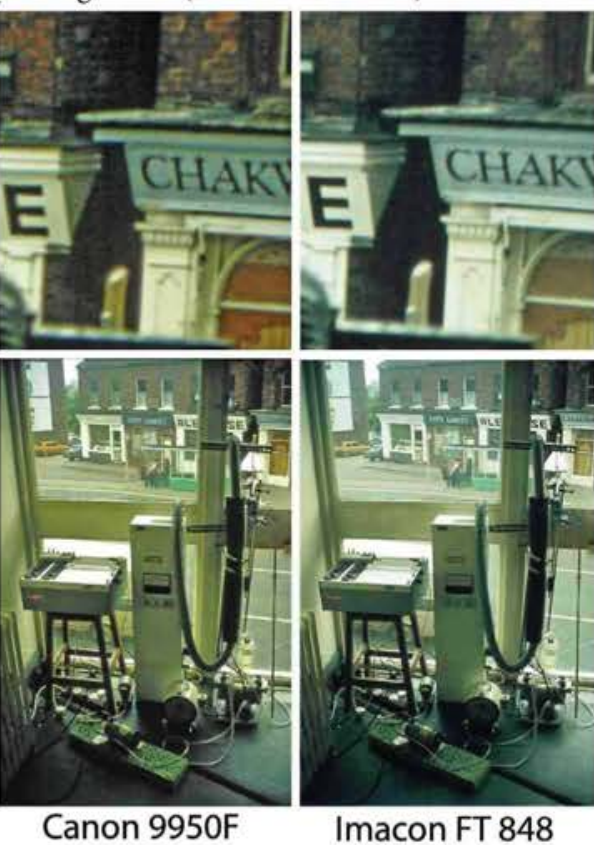

Figure 4. Scanned images of $35 \mathrm{~mm} \mathrm{Agfa}$ CS (ASA 100) slide film by the Canon $9950 \mathrm{~F}$ flatbed at 4,800 dpi and the Imacon Flextight 848 at 8,000 dpi. The photograph was taken with a consumer quality SLR in 1979. The image had considerable shadow on the sampling equipment, which was resolved by both scanners, although the Canon $9950 \mathrm{~F}$ image needed post-scan image shadow enhancement in Photoshop CS. An area has been enlarged from the shops seen outside the open window to demonstrate the scan resolution. The unedited Flextight scans were closer to that of the original slide in terms of colour and brightness (if a little overbright). 


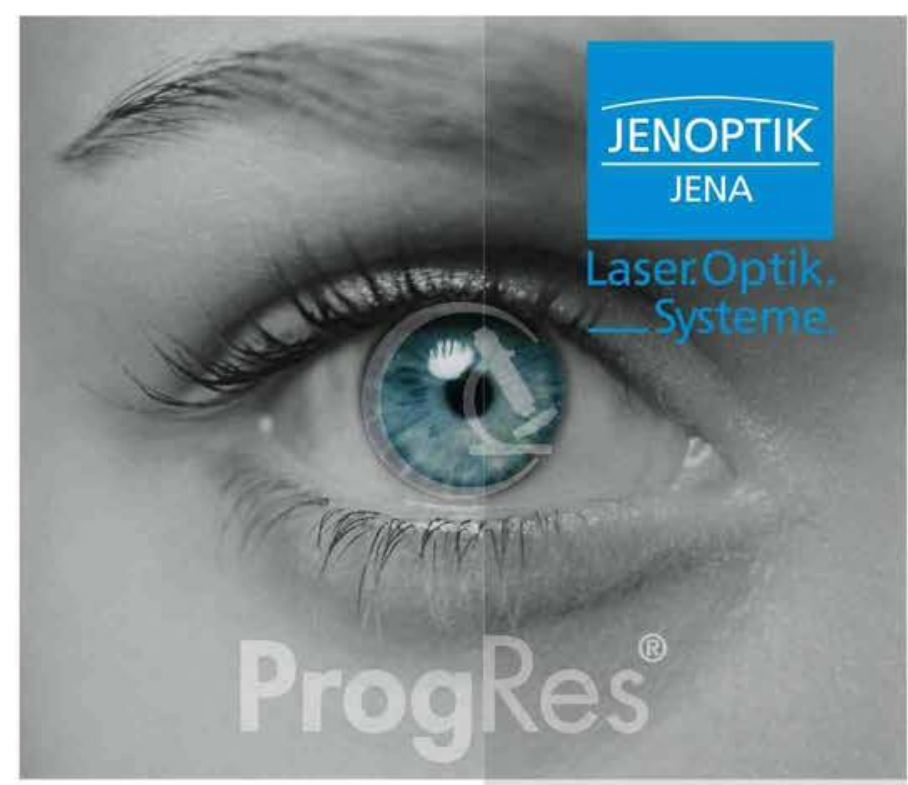

ProgRes ${ }^{\circledR}$ cameras as digital turnkey-solution

Routine cameras for analysis and documentation High-resolution cameras for scientific research Cooled monochrome cameras for fluorescence

In combination with easylab image management system from IMAGIC AG

ProgRes ${ }^{\text {क }}$ cameras are the basis for a turnkey-solution for processing, documentation and presentation of high-quality digital images increasing the efficiency of your lab.

Further information: www.progres-camera.com

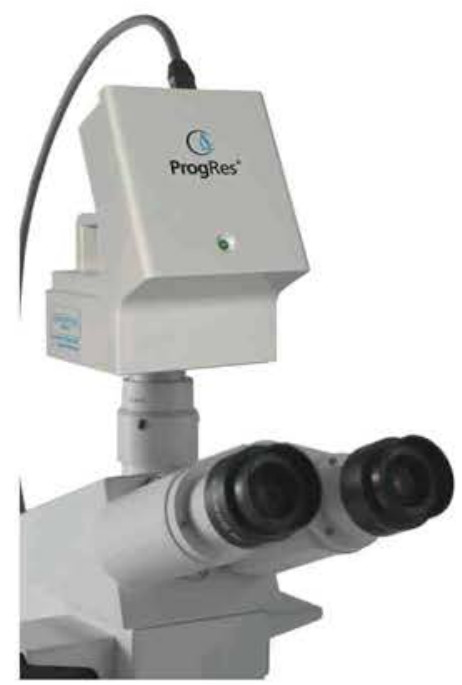

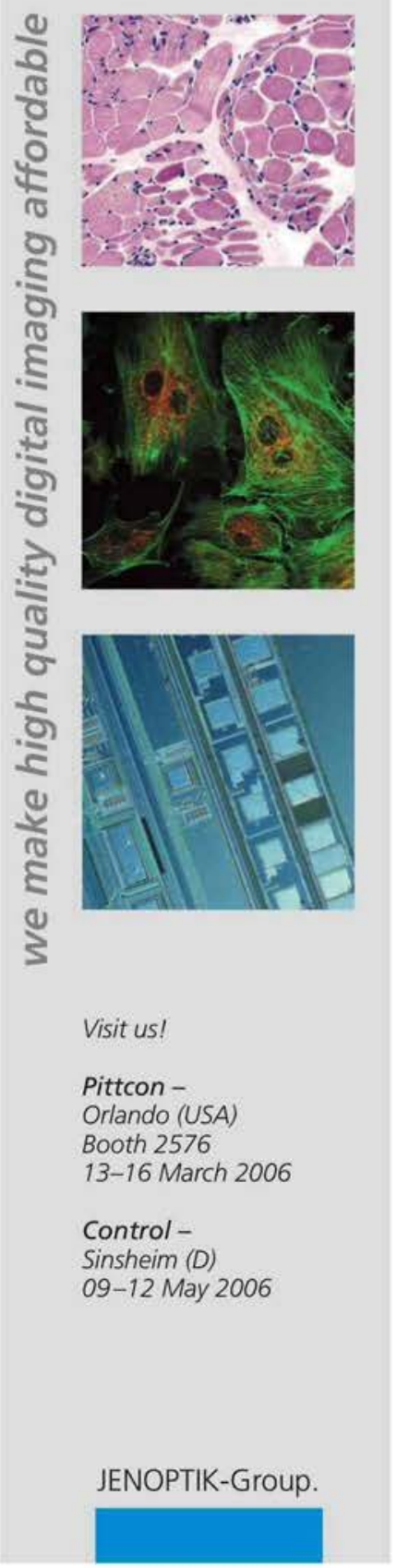

\section{INTBODUGING} The New High-fesolution Turbo Sputter Coater

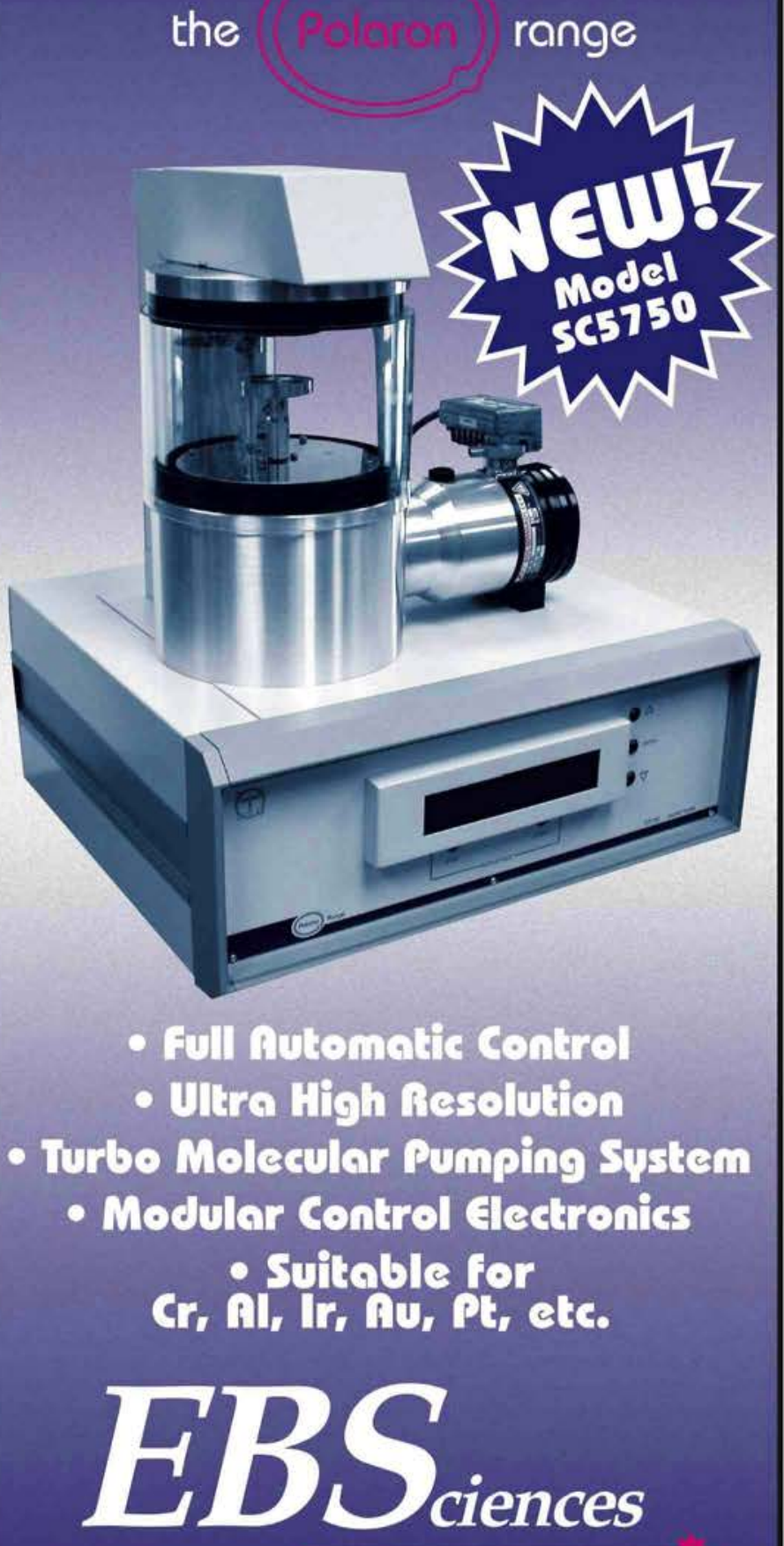

ADDING BRILLIANCE TO YOUR VISION

800-992-9037 or 860-635-0411 e-mail: cbs@ebsciences.com www.ebsciences.com 
was no doubt colour corrected, whereas Canon and Epson flatbeds weren't. The Flextight 848 scans looked most like the original slides in terms of colour and shadows, although there was no real difference in resolution. In fact, the darker saturated colour of the Canon $9950 \mathrm{~F}$ scans provided slightly more detail if anything. Note that all colour and B\&W film scans made with the comparably priced Epson 4990 Photo produced scans that were indistinguishable from those of the Canon 9950F. The Flextight drum scans had marginally more detail with very black regions of the TEM negative at $100 \%$ enlargement. There was some evidence of scanning degradation with all images, as a fraction more detail could been seen in the original slide film when viewed using a light box and magnifier.

Given the high resolution of the Kodak B\&W TEM film; it is perhaps surprising that the cheap flatbed scanners made such a good show of it, needing little or no post scan editing. Also it's rather unlikely many will want to archive TEM film digitally when the storage time of the original negative is 500 years or more, and the image file size would be well over $100 \mathrm{Mb}$ and highly prone to PC file corruption. Most will scan TEM film at 1,200 dpi or $800 \mathrm{dpi}$ for working copies for publication or image analysis and keep the original negative archived. Enlargements of selected areas may be scanned at 2,400 dpi. As no ICE or FARE dust removal was applied to the scans, all the cheaper flatbed scanners took around 2 to 4 minutes per scan.

Scanning graph paper on the cheap flatbed scanners demonstrated that there isn't really a 'sweet spot' on the glass bed, and distortion length errors were around $0.15 \%$ at $2,400 \mathrm{dpi}$ (rising to $0.3 \%$ at $800 \mathrm{dpi}$ ), when subsequently measured with a MetaMorph image analysis package (http://www. moleculardevices.com). Thus you can scan six TEM negatives in one go on the Epson flatbeds and the twain interface can be programmed to scan them independently. Older scanners such as the SCSI DuoScan T2550 had a special area on the film scanner bed (the size of one TEM negative), although, as can be seen in figure 3 , it produced no gain in scan quality. I did notice on one TEM scan that the cheap Epson 4990 Photo scanner slipped while scanning

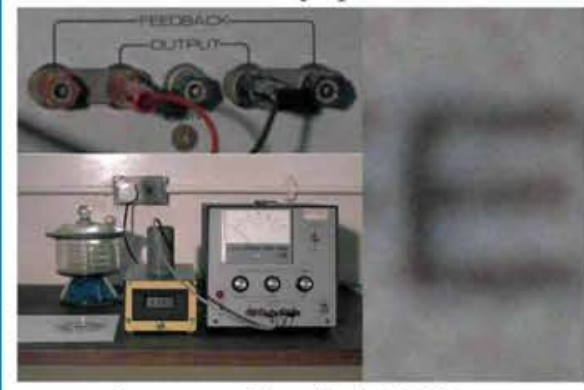

Imacon Flextight 848

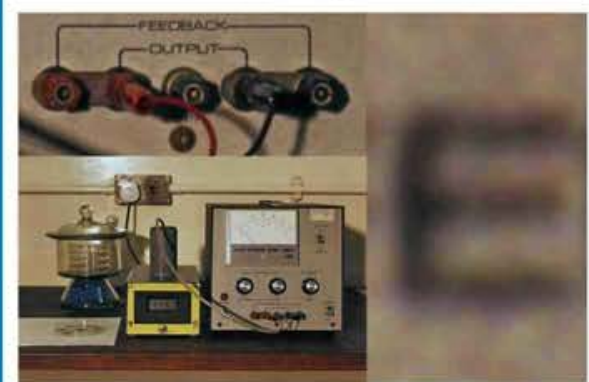

Canon 9950F

Figure 5. Indoor shot using flash of laboratory equipment, taken with a consumer quality SLR in the 1981, using Agfa CS (ASA 100) $35 \mathrm{~mm}$ slide film. The film was scanned at 4,800 dpi with the Canon 9950F flatbed and at 8,000 dpi with the Imacon Flextight 848. Again colour balance was more accurate with the Flextight, although there was little difference in resolution. Areas of the image were enlarged with the 'E' being the second letter in FEEDBACK on the power supply. increased PC file sizes by $4 \mathrm{x}$. If you are using modern very high resolution $35 \mathrm{~mm}$ colour film with a professional SLR film camera, you may get better scans with a dedicated $35 \mathrm{~mm}$ slide/film scanner or pro scanner, and the scan images will need less Photoshop editing afterwards. However if you are scanning large format film such as $70 \mathrm{~mm}, 1 / 4$ plate TEM and 120 , these cheap flatbed scanners give great results. Those who wish to archive large numbers of films with the highest resolution will still probably prefer to keep to their drum scanners, many of which have automated film handling and fast scanning speeds. For example the Flextight 848 can take 10 film holders (60 negatives or slides) in a single automatic batch, compared to the just eight on the platter with the Epson 4990 or twelve with the Canon 9950F and Epson V700 \& V750. A flatbed like the $\$ 45,000$ Kodak EverSmart Supreme II can scan 48 slides on it's A3 flatbed in one go, at 120 slides per hour (compared to about 12 per hour using a Canon $9950 \mathrm{~F}$ flatbed). Expensive scanners will also probably survive long scan runs better, although a maintenance contract on an Imacon Flextight 848 will be around $\$ 1,000$ per year.

So for film scanning on a budget the new Epson V700 or V750 Pro (even better than the Canon 9950F and the Epson 4990 Photo) would seem a very good choice. These two new scanners may even kill off dedicated $35 \mathrm{~mm}$ slide scanners (www.photo-i.co.uk). Few dedicated slide scanners can go beyond 9x6cm film size, and certainly can't match the Epson's V750, V700 and 4990 Photo's) ability to scan negative film and reflective photographs up to A4. The Canon $9950 \mathrm{~F}$ is limited to it's film holder sizes though, and lacks the flexible A4 film scanning of the Epson's using it's twain interface (plus Silverfast Ai supports Epson's ICE but not Canon's FARE dust removal). Naturally, these cheap flatbeds offer superb reflective scanning of photographs and documents as well. So whatever your budget there are now some great versatile film scanners around, for considerably less cost than they used to be.

\section{References}

The internet is a very valuable source of information on photography and associated activities like scanning film. Most of the information is free to view, being provided by photography enthusiasts or scientific establishments. Below is a small selection of good photographic sites. Much of the information in this article relies heavily on these sites.

http://www.photoscientia.co.uk/Grain.htm for discussions of grain size.

http://www.datamind.co.uk/merchant/resolution.htm for some discussion of pixel and image file sizes.

http://www.earthboundlight.com/phototips.html Bob Johnsons photographic pages.

http://www.thepluginsite.com/products/photowiz/index.htm The Photowiz website that offers USM plug-ins for Photoshop.

http://www.ted.photographer.org.uk/photoscience_colour.htm Ted's photographs, for discussions of colour photography.

http://www.mrothery.co.uk/vision/EyeNotes.htm The eye and colour perception. http://www.colour.org.uk/ physics of colour.

http://www.screensound.gov.au Film and archiving.

http://www.tasi.ac.uk/advice/creating/colour2.html working with colour in print.

http://www.cee.hw.ac.uk/hipr/html/gryimage.html greyscale information for B\&W images.

http://www.scantips.com A lot of good scanning tips by Wayne Fulton. Also available as book.http://www.kenrockwell.com/tech/scantek.htm Ken Rockwel scanning information.

http://medfmt.8k.com/mf/lenslpm.html Discussions on film resolution.

http://www.silverfast.com Producers of independent twain software for most scan ners. Often supplied with the more expensive film scanners.

www.kodak.com Now the suppliers of Digital ICE, SHO, ROC \& GEM, as well as the Creo range of large professional flatbed film scanners.

http://www.photo-i.co.uk Independent reviews of prosumer film scanners.

http://www.dpreview.com Independent reviews of digital cameras.

http://www.flatbed-scanner-review.org Pay per view scanner reviews for the professional.

http://www.pcpro.co.uk and http://www.computershopper.co.uk PC magazines provide many useful free reviews of cameras and scanners.

http://www.realviz.com/photo_stitching_software.htm Photo-stitching software: stitcher 4 for merging multiple images, e.g. multiple TEM fields, into one large image. Also used to create panarama's from multiple camera shots. Adobe Photoshop has the Photomerge application, but many free stitching utilities that come with digital cameras work better. 


\section{Multiphase Materials Characterization Fully Integrated...Totally Seamless}

Powerful Analytical Tools Optimized for Your SEM to Provide Results with Confidence

- Pegasus...Simultaneous data acquisitions for EDS (chemistry) and EBSD (crystallography)Includes the patented Chl-Scan software to improve multiphase analysis capability

- Neptune...Complimentary union of EDS and WDS for superior X-ray microanalysis-Improves quantification especially for low level concentrations

- Trident...Ultimate materials characterization system integrates EDS, EBSD, and WDS with no analytical performance compromise-Provides complete solutions for all your materials characterization requirements

\section{Results with Confidence}

As the world's leader in Electron Beam Microanalysis, EDAX continues to raise the performance standard with innovative features that are easy to adapt and provides results that you can trust to be complete and accurate.

For more information on our Seamless Integrated Systems for $E D S, E B S D$, and WDS visit our web site at www.EDAX.com/integration or call 1.201.529.4880 See us at PITTCON booth 3555!

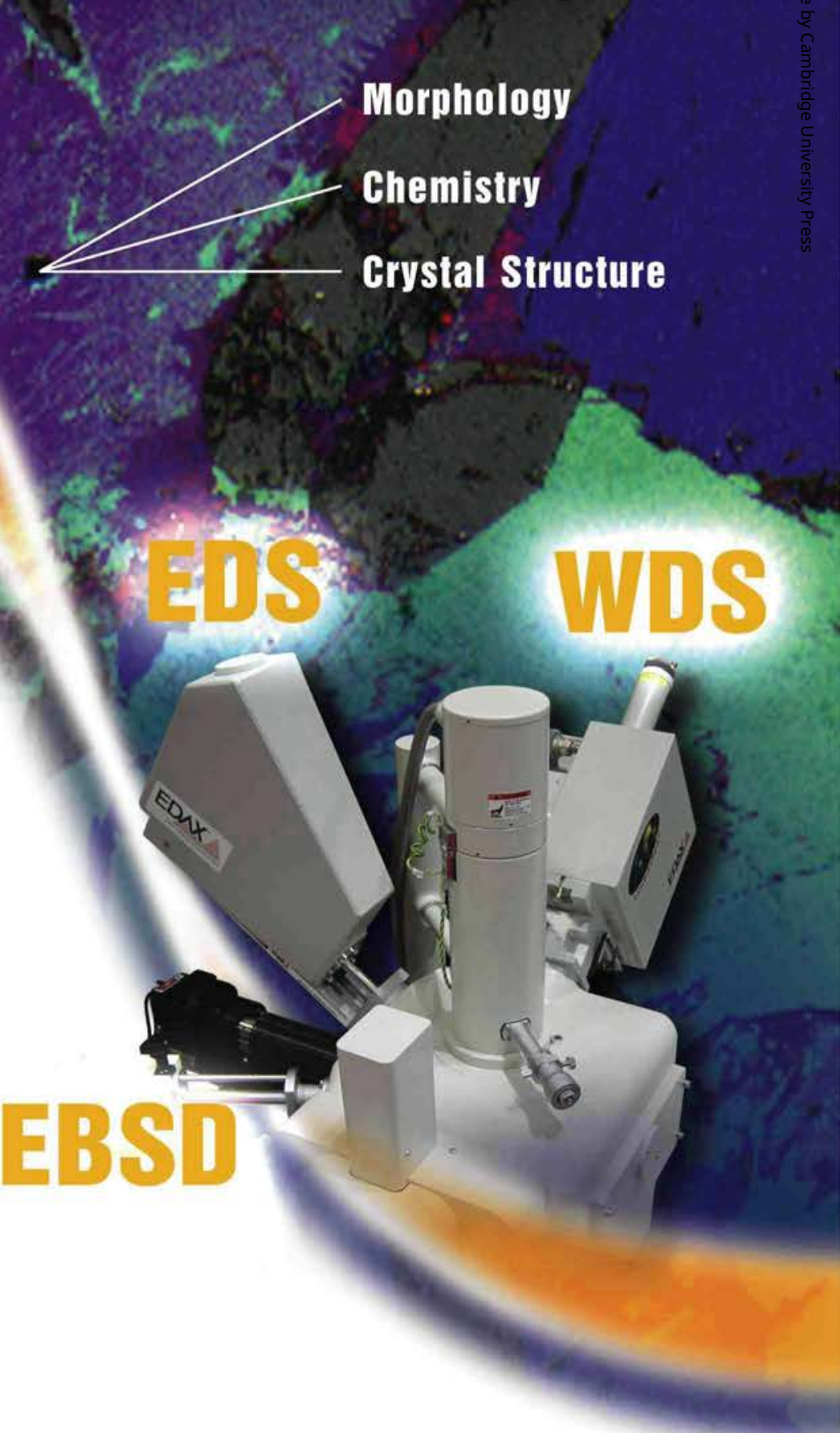

